\title{
PEALD of $\mathrm{HfO}_{2}$ thin films: Precursor tuning and a new near ambient pressure XPS approach to in-situ examination of thin film surfaces exposed to reactive gases
}

David Zanders ${ }^{1}$, Engin Ciftyurek ${ }^{2}$, Ersoy Subasi ${ }^{3}$, Niklas Huster ${ }^{1}$, Claudia Bock ${ }^{4}$, Aleksander Kostka ${ }^{5}$, Detlef Rogalla ${ }^{6}$, Klaus Schierbaum ${ }^{2}$ and Anjana Devi ${ }^{*}{ }^{*}$

${ }^{1}$ Inorganic Materials Chemistry, Ruhr University Bochum, 44801 Bochum, Germany.

${ }^{2}$ Department of Materials Science, Institute of Experimental Physics and Condensed Matter, HeinrichHeine-University Düsseldorf, 40225 Düsseldorf, Germany.

${ }^{3}$ Electronic Materials and Nanoelectronics, Ruhr University Bochum, 44801 Bochum, Germany.

${ }^{4}$ Microsystems Technology, Ruhr University Bochum, 44801, Germany.

${ }^{5}$ Center for Interface Dominated Materials (ZGH), Ruhr University Bochum, 44801, Germany.

${ }^{6}$ RUBION, Ruhr University Bochum, 44801, Germany.

${ }^{*}$ Corresponding Author: anjana.devi@rub.de 


\section{Synthesis of parental amides [Hf(NEtMe) $\left.)_{4}\right]$ and $\left[\mathrm{Hf}\left(\mathrm{Net}_{2}\right)_{4}\right]$ :}

The parental tetrakis-dialkylamido Hf compounds [Hf(NEtMe $\left.)_{4}\right]$ and $\left[\mathrm{Hf}\left(\mathrm{NEt}_{2}\right)_{4}\right]$ were synthesized via salt-metathesis reaction of in-situ prepared lithium dialkylamides and $\mathrm{HfCl}_{4}$ purchased by Alfa Aesar. Hereby, lithiation of secondary amines HNEtMe and HNEt2 (both Sigma Aldrich) was performed under cooling in diethyl ether by addition of $1.6 \mathrm{M} \mathrm{n-}$ butyl lithium solution (Sigma Aldrich). Both starting reagents were synthesized in large batches $(\sim 10$ g) with yields typically ranging between (85 - 90) \%. Prior to use, the purity of the compounds was assessed by nuclear magnetic resonance spectroscopic methods ( $\left.{ }^{1} \mathrm{H}-\mathrm{NMR},{ }^{13} \mathrm{C}-\mathrm{NMR}\right)$ and elemental analysis (EA). [Hf(NEtMe) 4 : ${ }^{\mathbf{1}} \mathbf{H}-\mathrm{NMR}(250 \mathrm{MHz}$, Benzene- $\left.\mathrm{d}_{6}\right): \delta(\mathrm{ppm})=3.29\left(\mathrm{q}, \mathrm{J}=6.98 \mathrm{~Hz}, 8 \mathrm{H}, \mathrm{N}-\mathrm{CH}_{2}-\mathrm{CH}_{3}\right), 3.00\left(\mathrm{~s}, 12 \mathrm{H}, \mathrm{N}-\mathrm{CH}_{3}\right), 1.17\left(\mathrm{t}, \mathrm{J}=6.96 \mathrm{~Hz}, 12 \mathrm{H}, \mathrm{N}-\mathrm{CH}_{2}-\mathrm{CH}_{3}\right)$;

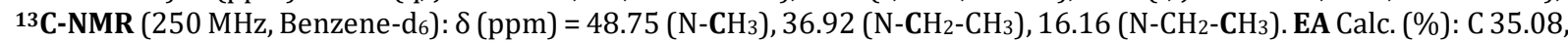
H 7.85, N 13.64; Found (\%): C 34.90, H 7.63, N 13.27. [Hf(NEt2)4]: ${ }^{1}$ H-NMR (250 MHz, Benzene-d6): $\delta(p p m)=3.37$ (q, J $\left.=6.96 \mathrm{~Hz}, 16 \mathrm{H}, \mathrm{N}-\mathrm{CH}_{2}-\mathrm{CH}_{3}\right), 1.16\left(\mathrm{t}, \mathrm{J}=6.96 \mathrm{~Hz}, 24 \mathrm{H}, \mathrm{N}-\mathrm{CH}_{2}-\mathrm{CH}_{3}\right) ;{ }^{13} \mathrm{C}-\mathrm{NMR}\left(250 \mathrm{MHz}\right.$, Benzene- $\left.\mathrm{d}_{6}\right): \delta(\mathrm{ppm})=42.93$ $\left(\mathrm{N}-\mathrm{CH}_{2}-\mathrm{CH}_{3}\right), 16.32\left(\mathrm{~N}-\mathrm{CH}_{2}-\mathrm{CH}_{3}\right)$. EA Calc. (\%): C 41.15, H 8.63, N 12.00; Found (\%): C 41.16, H 8.89, N 12.11.

Scheme S1: Optimized pulse/purge sequences for the PEALD process between $60^{\circ} \mathrm{C}$ and $240{ }^{\circ} \mathrm{C}$.

\section{Pulse \& Purge Sequence for PEALD depositions}

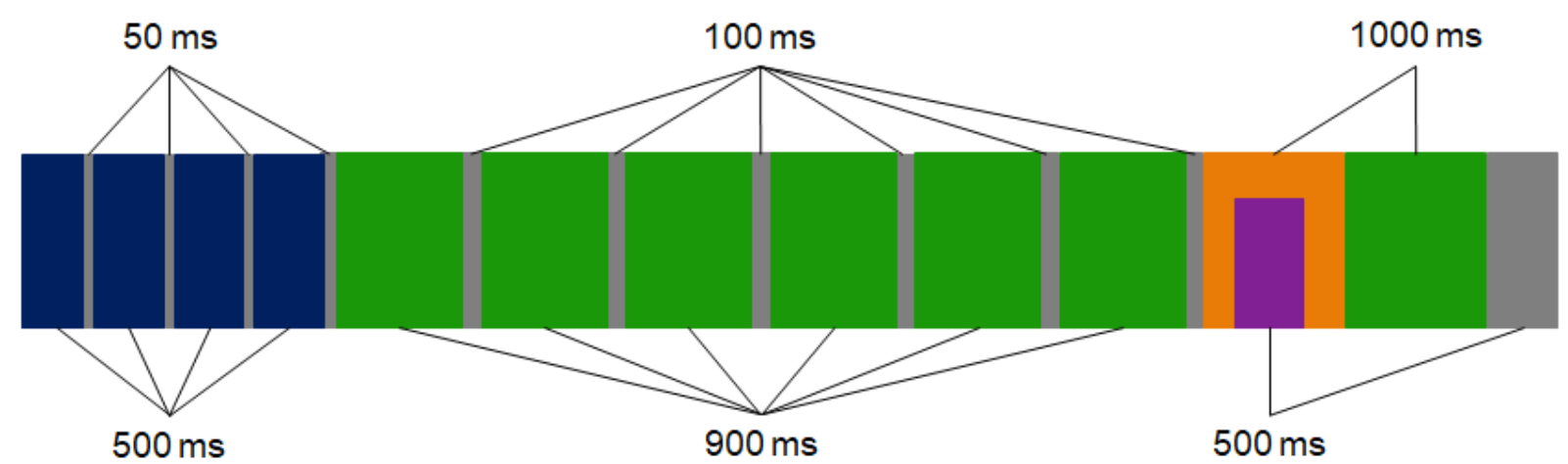

Legend:

Precursor pulse

Vacuum

Argon pulse

Oxygen pulse

Plasma pulse

\section{Mass spectrometric data for compounds (1) - (4):}

$\left[\mathrm{Hf}\left\{\eta^{2}-\left(\mathrm{PrN}_{2} \mathrm{CNEtMe}\right\}(\mathrm{NEtMe})_{3}\right](\mathbf{1})\right.$ :

EI-MS $(70 \mathrm{eV}) \mathrm{m} / \mathrm{z}(\%)$ [fragment]: $538.4(16.9 \%)[\mathrm{M}]^{+}, 480.4(53.4 \%)[\mathrm{M}-\mathrm{L}]^{+}, 478.3(71.7 \%)[\mathrm{M}-4 \mathrm{x} \mathrm{Me}]^{+}, 464.3$ $(7.3 \%)[\mathrm{M}-\mathrm{Et}-3 \mathrm{x} \mathrm{Me}]^{+}, 450.3(2.6 \%)[\mathrm{M}-\mathrm{L}-2 \mathrm{x} \mathrm{Me}]^{+}, 436.3(16.6 \%)[\mathrm{M}-\mathrm{L}-\mathrm{Et}-\mathrm{Me}]^{+}, 422.3(16.9 \%)[\mathrm{M}-2 \mathrm{x} \mathrm{L}]^{+}$, $421.3(28.5 \%)$ [M - L - Et - 2x Me] $]^{+}, 393.2$ (8.6\%) [M - 2x L - Et] ${ }^{+}, 377.2$ (4.3\%) [M - 2x L - Et - Me] $]^{+}, 364.2(3.1 \%)[\mathrm{M}-$ $3 \mathrm{x} \mathrm{L}]^{+}, 350.2(23.0 \%)[\mathrm{M}-3 \mathrm{x} \mathrm{L}-\mathrm{Me}]^{+}, 334.2(12.8 \%)[\mathrm{M}-3 \mathrm{x} \mathrm{L}-2 \mathrm{x} \mathrm{Me}]^{+}, 320.1(6.2 \%)\left[\mathrm{M}-3 \mathrm{x} \mathrm{L}-\mathrm{i}_{\mathrm{Pr}}\right]^{+}, 307.2(17.1 \%)$ [M - 3x L - iPr - Me]+, 291.1 (13.4\%) [M - 3x L - iPr - 2x Me $]^{+}, 277.1$ (4.6\%) [M - 3x L - 2x $\left.{ }^{i} \mathrm{Pr}\right]^{+}, 263.1$ (5.5 \%) [M - 3x L $\left.2 \mathrm{x}^{\mathrm{i}} \mathrm{Pr}-\mathrm{Me}\right]^{+}, 249.0$ (4.3\%) [M - 3x L - 2x $\left.\mathrm{Pr}-\mathrm{Et}\right]^{+}, 234.1(1.7 \%)[\mathrm{M}-3 \mathrm{x} \mathrm{L}-2 \mathrm{x} \text { iPr }-\mathrm{Et}-\mathrm{Me}]^{+}, 220.1(0.6 \%)[\mathrm{M}-4 \mathrm{x} \mathrm{L}-$ $\left.2 \mathrm{x}^{\mathrm{i} P r}\right]^{+}, 185.2(13.8 \%)[\mathrm{GH}]^{+}, 170.2(5.6 \%)[\mathrm{GH}-\mathrm{Me}]^{+}, 143.2(0.9 \%)\left[\mathrm{GH}-\mathrm{C}_{3} \mathrm{H}_{6}\right]^{+}, 128.1(25.8 \%)\left[\mathrm{GH}-\mathrm{C}_{3} \mathrm{H}_{6}-\mathrm{Me}^{+}\right.$, 113.1 (26.9 \%) [GH - $\left.\mathrm{C}_{3} \mathrm{H}_{6}-2 \mathrm{x} \mathrm{Me}\right]^{+}, 85.1$ (53.3\%) [GH - $\left.\mathrm{C}_{3} \mathrm{H}_{6}-\mathrm{L}\right]^{+}, 59.1(34.6 \%)[\mathrm{LH}]^{+}, 58.1(68.4 \%)[\mathrm{L}]^{+}, 44.1(100 \%)$ $[\mathrm{LH}-\mathrm{Me}]^{+}, 43.1(75.4 \%)[\mathrm{L}-\mathrm{Me}]^{+}, 42.1(34.8 \%)\left[\mathrm{C}_{3} \mathrm{H}_{6}\right]^{+}, 30.0(40.2 \%)[\mathrm{LH}-\mathrm{Et}]^{+}, 29.1(17.4 \%)[\mathrm{L}-\mathrm{Et}]^{+}, 28.0(37.8 \%)$ $\left[\mathrm{C}_{2} \mathrm{H}_{4}\right]^{+}, 26.0(6.2 \%)\left[\mathrm{C}_{2} \mathrm{H}_{2}\right]^{+}, 15.0(15.4 \%)\left[\mathrm{CH}_{3}\right]^{+}$. With: $\mathrm{M}^{+}=$Molecular ion, $\mathrm{G}=$ Guanidinato ligand, $\mathrm{L}=$ Alkylamide ligand, $\mathrm{H}=$ Hydrogen.

$\left[\mathrm{Hf}\left\{\eta^{2}-(\mathrm{PrN})_{2} \mathrm{CNEt}_{2}\right\}\left(\mathrm{NEt}_{2}\right)_{3}\right](2)$ :

EI-MS $(70 \mathrm{eV}) \mathrm{m} / \mathrm{z}(\%)$ [fragment]: $594.5(5.5 \%)[\mathrm{M}]^{+}, 522.4(88.3 \%)[\mathrm{M}-\mathrm{L}]^{+}, 520.4$ (71.7 \%) [M - 4x Et] $]^{+}, 492.4$ $(11.8 \%)[\mathrm{M}-\mathrm{L}-2 \mathrm{x} \mathrm{Me}]^{+}, 478.4$ (5.6\%) [M - L - Et - Me]+, $465.3(12.4 \%)[\mathrm{M}-\mathrm{L}-2 \mathrm{x} \mathrm{Et}]^{+}, 450.4(19.6 \%)[\mathrm{M}-2 \mathrm{x} \mathrm{L}]^{+}$, $449.3(28.0 \%)[\mathrm{M}-\mathrm{L}-2 \mathrm{x} \mathrm{Et}-\mathrm{Me}]^{+}, 421.3(17.0 \%)[\mathrm{M}-2 \mathrm{x} \mathrm{L}-\mathrm{Et}]^{+}, 405.3(3.4 \%)[\mathrm{M}-2 \mathrm{x} \mathrm{L}-3 \mathrm{x} \mathrm{Me}]^{+}, 392.3(8.8 \%)[\mathrm{M}-$ $2 \mathrm{x} \mathrm{L}-2 \mathrm{x} \mathrm{Et}]^{+}, 378.2(11.2 \%)[\mathrm{M}-3 \mathrm{x} \mathrm{L}]^{+}, 362.2(4.8 \%)[\mathrm{M}-3 \mathrm{x} \mathrm{L}-\mathrm{Me}]^{+}, 348.2(4.4 \%)[\mathrm{M}-3 \mathrm{x} \mathrm{L}-2 \mathrm{x} \mathrm{Me}]^{+}, 335.2(11.3 \%)$ $\left[\mathrm{M}-3 \mathrm{x} \mathrm{L}-{ }^{\mathrm{i} P r}\right]^{+}, 321.1$ (6.8\%) [M - 3x L - i Pr - Me]+, 305.2 (5.6\%) [M - 3x L - i Pr - 2x Me] $]^{+}, 292.1$ (2.3 \%) [M - 3x L - 2x 
$\left.{ }^{i} \mathrm{Pr}\right]^{+}, 277.8(4.2 \%)[\mathrm{M}-3 \mathrm{x} \mathrm{L}-2 \mathrm{x} \text { iPr }-\mathrm{Me}]^{+}, 263.1$ (3.3\%) [M - 3x L - 2x $\left.{ }^{\mathrm{i} P r}-\mathrm{Et}\right]^{+}, 248.1(1.7 \%)\left[\mathrm{M}-3 \mathrm{x} \mathrm{L}-2 \mathrm{x}{ }^{\mathrm{i}} \mathrm{Pr}-\mathrm{Et}-\right.$ $\mathrm{Me}]^{+}, 234.1(0.9 \%)[\mathrm{M}-3 \mathrm{x} \mathrm{L}-2 \mathrm{x} \text { iPr }-2 \mathrm{x} \mathrm{Et}]^{+}, 220.1(0.2 \%)\left[\mathrm{M}-4 \mathrm{x} \mathrm{L}-2 \mathrm{x}^{\mathrm{i}} \mathrm{Pr}\right]^{+}, 199.2(0.1 \%)[\mathrm{GH}]^{+}, 157.2(0.2 \%)[\mathrm{GH}$ $\left.-\mathrm{C}_{3} \mathrm{H}_{6}\right]^{+}, 141.2\left(4.3 \% \text { ) [G }-\mathrm{C}_{3} \mathrm{H}_{6}-\mathrm{Me}\right]^{+}, 127.1$ (3.1\%) [GH - $\left.\mathrm{C}_{3} \mathrm{H}_{6}-2 \mathrm{x} \mathrm{Et}\right]^{+}, 99.1$ (18.3\%) [G - 2x C $\left.\mathrm{C}_{3} \mathrm{H}_{6}-\mathrm{Me}\right]^{+}, 72.1(7.6 \%)$ $[\mathrm{L}]^{+}, 58.1(6.5 \%)[\mathrm{LH}-\mathrm{Me}]^{+}, 43.1(18.9 \%)[\mathrm{L}-\mathrm{Et}]^{+}, 42.1(2.4 \%)\left[\mathrm{C}_{3} \mathrm{H}_{6}\right]^{+}, 29.1(4.5 \%)[\mathrm{LH}-\mathrm{Et}-\mathrm{Me}]^{+}, 28.0(2.0 \%)$ $\left[\mathrm{C}_{2} \mathrm{H}_{4}\right]^{+}, 15.0(0.6 \%)\left[\mathrm{CH}_{3}\right]^{+}$.

\section{$\left[\mathrm{Hf}\left\{\eta^{2}-((\mathrm{EtN})(\mathrm{tBuN})) \mathrm{CNEtMe}\right\}(\mathrm{NEtMe})_{3}\right](3):$}

EI-MS $(70 \mathrm{eV}) \mathrm{m} / \mathrm{z}(\%)$ [fragment]: 538.6 (8.8 \%) [M] $]^{+}, 480.3(64.3 \%)[\mathrm{M}-\mathrm{L}]^{+}, 478.5$ (64.4 \%) [M - 4x Me]+, 465.5 $(2.7 \%)[\mathrm{M}-\mathrm{L}-\mathrm{Me}]^{+}, 464.4(15.9 \%)[\mathrm{M}-\mathrm{Et}-3 \mathrm{x} \mathrm{Me}]^{+}, 450.4(2.2 \%)[\mathrm{M}-\mathrm{L}-2 \mathrm{x} \mathrm{Me}]^{+}, 422.4(36.0 \%)[\mathrm{M}-2 \mathrm{x} \mathrm{L}]^{+}, 421.4$ $(100 \%)[\mathrm{M}-\mathrm{L}-\mathrm{Et}-2 \mathrm{x} \mathrm{Me}]^{+}, 407.4$ (7.5\%) [M - 2x L - Me]+, $393.3(7.0 \%)[\mathrm{M}-2 \mathrm{x} \mathrm{L}-\mathrm{Et}]^{+}, 378.3(12.3 \%)[\mathrm{M}-2 \mathrm{x} \mathrm{L}-\mathrm{Et}-$ $\mathrm{Me}]^{+}, 364.3(7.0 \%)[\mathrm{M}-3 \mathrm{x} \mathrm{L}]^{+}, 350.3(30.0 \%)[\mathrm{M}-3 \mathrm{x} \mathrm{L}-\mathrm{Me}]^{+}, 336.3(29.2 \%)[\mathrm{M}-3 \mathrm{x} \mathrm{L}-\mathrm{Et}]^{+}, 334.2(27.5 \%)[\mathrm{M}-3 \mathrm{x} \mathrm{L}$ - 2x Me] ${ }^{+}, 320.2$ (14.9\%) [M - 3x L - Et - Me]+ 305.2 (13.4\%) [M - 3x L - Et - 2x Me]+, 290.2 (12.9\%) [M - 3x L - Et - 3x $\mathrm{Me}]^{+}, 278.2$ (5.4\%) [M - 3x L - Et - tBu], 263.2 (9.8\%) [M - 3x L - Et - tBu - Me] ${ }^{+}, 250.0$ (6.5 \%) [M - 3x L - 2x Et - tBu] , $234.1(3.2 \%)[\mathrm{M}-3 \mathrm{x} \mathrm{L}-2 \mathrm{x} \mathrm{Et}-\mathrm{Me}-\mathrm{tBu}]^{+}, 220.1(1.0 \%)[\mathrm{M}-4 \mathrm{x} \mathrm{L}-\mathrm{Et}-\mathrm{tBu}]^{+}, 185.3(0.1 \%)[\mathrm{GH}]^{+}, 113.2(16.4 \%)$ [GH $\left.-{ }^{\mathrm{t} B u}\right]^{+}, 112.1(23.8 \%)[\mathrm{G}-\mathrm{tBu}]^{+}, 85.1(13.2 \%)\left[\mathrm{GH}-\mathrm{N}^{\mathrm{t} B u}-\mathrm{Et}\right]^{+}, 83.1(18.3 \%)\left[(\mathrm{C}) \mathrm{N}^{\mathrm{tBu}}\right]^{+}, 71.1(8.7 \%)\left[\mathrm{N}^{\mathrm{tBu}}\right]^{+}, 59.1$ $(4.9 \%)[\mathrm{LH}]^{+}, 58.1(16.0 \%)[\mathrm{L}]^{+} /$[tBu $^{+}, 57.1(58.4 \%)[\mathrm{CNEt}]^{+}, 56.1(7.9 \%)\left[\mathrm{NtBu}^{\mathrm{t}} \mathrm{Me}\right]^{+}, 44.1(18.3 \%)[\mathrm{LH}-\mathrm{Me}]^{+}$, $43.1(7.2 \%)[\mathrm{L}-\mathrm{Me}]^{+}, 41.1(20.1 \%)[\mathrm{CNMe}]^{+}, 29.1(16.5 \%)[\mathrm{L}-\mathrm{Et}]^{+}, 28.0(7.0 \%)\left[\mathrm{C}_{2} \mathrm{H}_{4}\right]^{+}, 15.0(3.4 \%)\left[\mathrm{CH}_{3}\right]^{+}$.

$\left[\mathrm{Hf}\left\{\eta^{2}-\left((\mathrm{EtN})\left({ }^{\mathrm{tBuN}}\right)\right) \mathrm{CNEt}_{2}\right\}\left(\mathrm{NEt}_{2}\right)_{3}\right](4)$ :

EI-MS $(70 \mathrm{eV}) \mathrm{m} / \mathrm{z}(\%)$ [fragment]: $594.6(2.8 \%)[\mathrm{M}]^{+}, 522.5(100 \%)[\mathrm{M}-\mathrm{L}]^{+}, 520.3(97.7 \%)$ [M - 4x Et] $]^{+}, 492.4(9.9 \%)$ $[\mathrm{M}-\mathrm{L}-2 \mathrm{x} \mathrm{Me}]^{+}, 466.4(24.4 \%)\left[\mathrm{M}-\mathrm{L}^{-\mathrm{tBu}}\right]^{+}, 464.4(18.6 \%)$ [M -L - 2x Et] $]^{+}, 450.4$ (28.5 \%) [M - 2x L]+, 449.4 (78.5\%) $[\mathrm{M}-\mathrm{L}-2 \mathrm{x} \mathrm{Et}-\mathrm{Me}]^{+}, 435.4(2.1 \%)[\mathrm{M}-2 \mathrm{x} \mathrm{L}-\mathrm{Me}]^{+}, 421.3(6.8 \%)[\mathrm{M}-2 \mathrm{x} \mathrm{L}-\mathrm{Et}]^{+}, 405.3$ (2.6\%) [M - 2x L - 3x Me]+, 392.3 (36.9\%) [M - 2x L - 2x Et] $]^{+}, 378.3(12.0 \%)[\mathrm{M}-3 \mathrm{x} \mathrm{L}]^{+}, 363.2(15.2 \%)[\mathrm{M}-3 \mathrm{x} \mathrm{L}-\mathrm{Me}]^{+}, 349.3$ (1.7 \%) [M - 3x L - Et] $]^{+}$, $348.2(5.0 \%)[\mathrm{M}-3 \mathrm{x} \mathrm{L}-2 \mathrm{x} \mathrm{Me}]^{+}, 334.2(13.4 \%)[\mathrm{M}-3 \mathrm{x} \mathrm{L}-\mathrm{Et}-\mathrm{Me}]^{+}, 319.2(12.2 \%)[\mathrm{M}-3 \mathrm{x} \mathrm{L}-\mathrm{Et}-2 \mathrm{x} \mathrm{Me}]^{+}, 304.2$ (5.8\%) [M - 3x L - Et - 3x Me $]^{+}, 292.2(4.6 \%)\left[\mathrm{M}-3 \mathrm{x} \mathrm{L}-\mathrm{Et}-\mathrm{tB}^{+}, 277.1(5.3 \%)[\mathrm{M}-3 \mathrm{x} \mathrm{L}-\mathrm{Et}-\mathrm{Me}-\mathrm{tBu}]^{+}, 263.1(6.1 \%)\right.$ [M - 3x L - 2x Et - $\left.{ }^{\mathrm{tBu}}\right]^{+}, 248.1$ (3.281\%) [M - 3x L - 3x Et - $\mathrm{tBu}^{+}, 220.1(0.8 \%)[\mathrm{M}-4 \mathrm{x} \mathrm{L}-\mathrm{Et}-\mathrm{t} \mathrm{Bu}]^{+}, 199.2(0.1 \%)[\mathrm{GH}]^{+}$, $127.2(17.3 \%)\left[\mathrm{GH}-\mathrm{Me}-\mathrm{t}^{\mathrm{Bu}}\right]^{+}, 111.1(12.4 \%)\left[\mathrm{G}-2 \mathrm{x} \mathrm{Me}-\mathrm{t}^{\mathrm{Bu}}\right]^{+}, 99.1(10.1 \%)\left[\mathrm{GH}-\mathrm{N}^{\mathrm{tB} u}\right]^{+}, 83.1(10.1 \%)\left[(\mathrm{C}) \mathrm{N}^{\mathrm{t} B u}\right]^{+}$, $\left.73.1(2.7 \%)[\mathrm{LH}]^{+}, 72.1(10.7 \%)[\mathrm{L}]^{+}, 71.1(10.7 \%)\left[\mathrm{N}^{\mathrm{t} B u}\right]^{+}, 58.1(15.1 \%)[\mathrm{LH}-\mathrm{Me}]^{+} /{ }^{\mathrm{t}} \mathrm{Bu}\right]^{+}, 57.1(36.0 \%)[\mathrm{L}-\mathrm{Me}]^{+}$, $56.1(13.1 \%)\left[\mathrm{NtBu}^{\mathrm{t}} \mathrm{Me}\right]^{+}, 44.1(5.7 \%)[\mathrm{LH}-\mathrm{Et}]^{+}, 43.1(7.2 \%)[\mathrm{L}-\mathrm{Et}]^{+}, 42.1(7.2 \%)[\mathrm{CHNMe}]^{+}, 41.1(14.0 \%)[\mathrm{CNMe}]^{+}$, $29.1(15.4 \%)[\mathrm{LH}-\mathrm{Et}-\mathrm{Me}]^{+}, 28.0(8.8 \%)\left[\mathrm{C}_{2} \mathrm{H}_{4}\right]^{+}, 15.0(2.5 \%)\left[\mathrm{CH}_{3}\right]^{+}$.
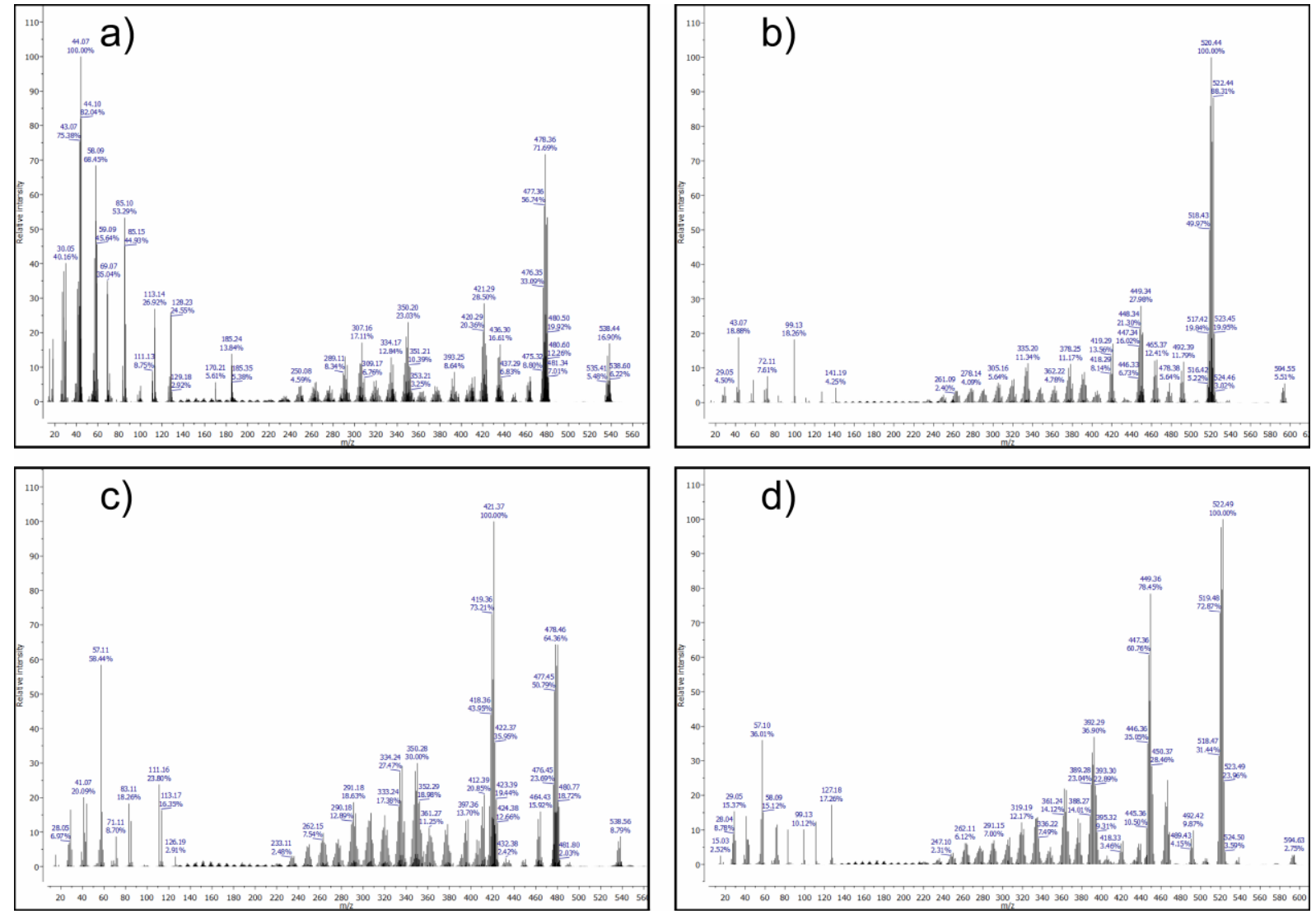

Figure S1: EI-MS spectra of compound (1) (a), (2) (b), (3) (c) and (4) (d). 
Table S1: Overview of selected, possible fragments found in EI-MS spectra of compounds (1) - (4).[a]

\begin{tabular}{|c|c|c|c|c|c|c|c|c|}
\hline Compound & \multicolumn{2}{|c|}{ (1) } & \multicolumn{2}{|c|}{ (2) } & \multicolumn{2}{|c|}{ (3) } & \multicolumn{2}{|c|}{ (4) } \\
\hline Fragment & $(\mathrm{m} / \mathrm{z})$ & $\begin{array}{l}\text { Rel. int. } \\
(\%)\end{array}$ & $(\mathrm{m} / \mathrm{z})$ & $\begin{array}{c}\text { Rel. int. } \\
(\%)\end{array}$ & $(m / z)$ & $\begin{array}{c}\text { Rel. int. } \\
(\%)\end{array}$ & $(\mathrm{m} / \mathrm{z})$ & $\begin{array}{c}\text { Rel. int. } \\
(\%)\end{array}$ \\
\hline $\mathrm{M}^{+}$ & 538.4 & 16.9 & 594.5 & 5.5 & 538.6 & 8.8 & 595.6 & 2.8 \\
\hline $\mathrm{M}^{+}-\mathrm{L}$ & 480.4 & 53.4 & 522.4 & 88.3 & 480.3 & 64.3 & 522.5 & 100 \\
\hline $\mathrm{M}^{+}-2 \mathrm{~L}$ & 422.3 & 16.9 & 450.4 & 19.6 & 422.4 & 36.0 & 450.4 & 28.5 \\
\hline$M^{+}-3 L$ & 364.2 & 3.1 & 378.1 & 11.2 & 364.3 & 7.0 & 378.3 & 12.0 \\
\hline $\mathrm{M}^{+}-3 \mathrm{~L}-\mathrm{Et}$ & - & - & - & - & 335.3 & 29.2 & 349.1 & 1.7 \\
\hline $\mathrm{M}^{+}-3 \mathrm{~L}-\mathrm{i} \mathrm{Pr}$ & 321.1 & 6.2 & 335.2 & 11.3 & - & - & - & - \\
\hline $\mathrm{M}^{+}-3 \mathrm{~L}-2^{\mathrm{i}} \mathrm{Pr}$ & 278.0 & 4.6 & 292.1 & 2.3 & - & - & - & - \\
\hline $\mathrm{M}^{+}-3 \mathrm{~L}-\mathrm{-}^{\mathrm{t}} \mathrm{Bu}-\mathrm{Et}$ & - & - & - & - & 278.2 & 5.4 & 292.2 & 4.6 \\
\hline $\begin{array}{c}\mathrm{M}^{+}-4 \mathrm{~L}-2^{\mathrm{i}} \mathrm{Pr} / \mathrm{M}^{+} \\
-4 \mathrm{~L}-\mathrm{B} \mathrm{Bu}-\mathrm{Et}\end{array}$ & 220.0 & 0.6 & 220.1 & 0.2 & 220.1 & 1.0 & 220.1 & 0.8 \\
\hline $\mathrm{L}^{+}$ & 58.1 & 68.4 & 72.1 & 7.6 & 58.1 & 16.0 & 72.1 & 10.7 \\
\hline
\end{tabular}

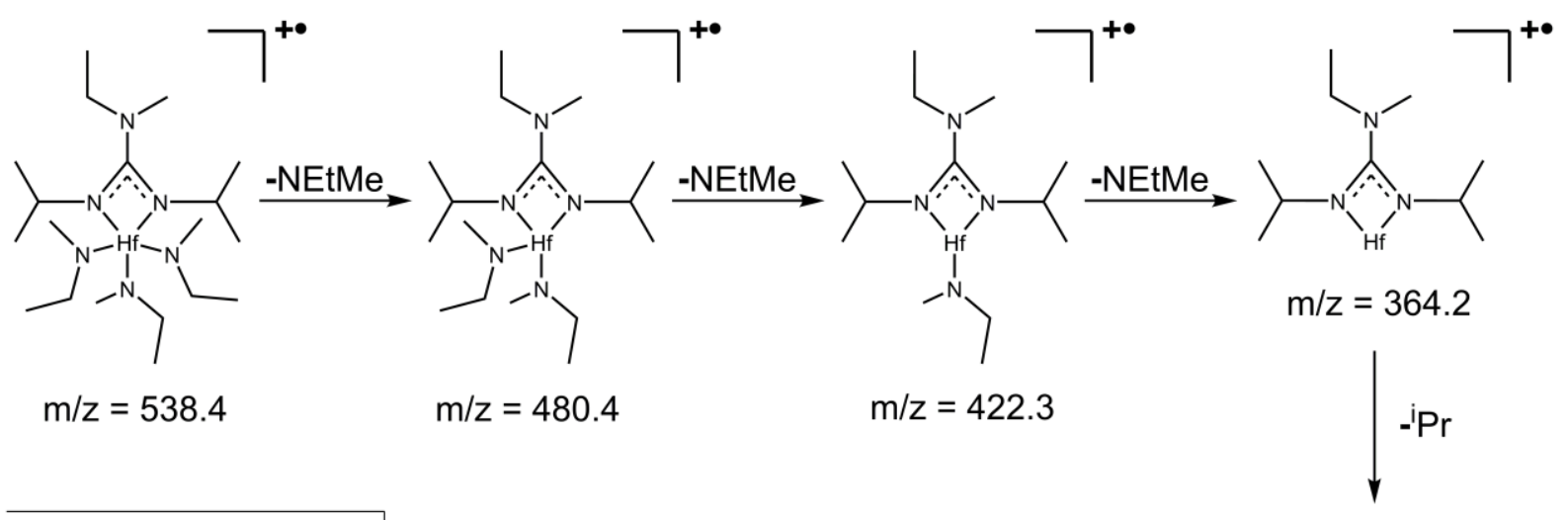

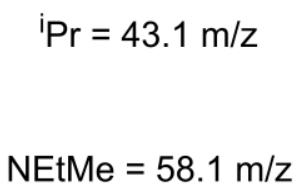

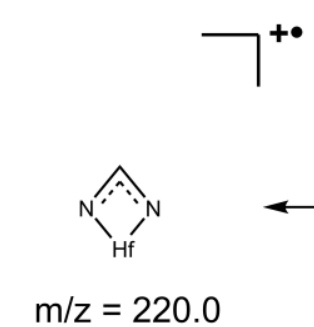

$\mathrm{m} / \mathrm{z}=220.0$
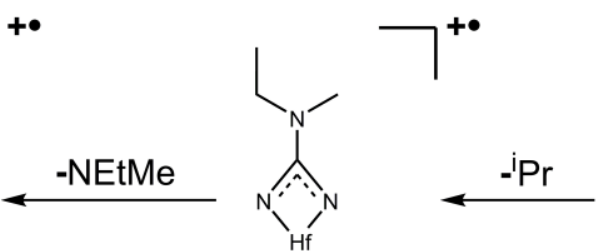

$\mathrm{m} / \mathrm{z}=278.0$

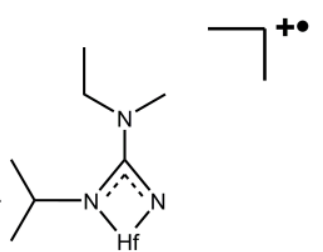

$\mathrm{m} / \mathrm{z}=321.1$

Figure S2: Proposed fragmentation pattern of synthesized Hf mono-guanidinato tris-alkylamide compounds (1)-(4) exemplified at hand of $\left[\mathrm{Hf}\left\{\eta^{2}-(\mathrm{PrN})_{2} \mathrm{CNEtMe}\right\}(\mathrm{NEtMe})_{3}\right](\mathbf{1})$ under mass spectrometric conditions (EI-MS, $\left.70 \mathrm{eV}\right)$. The preferential loss of alkylamide moieties is suggested by the respective $\mathrm{m} / \mathrm{z}$ ratios of the compounds. 

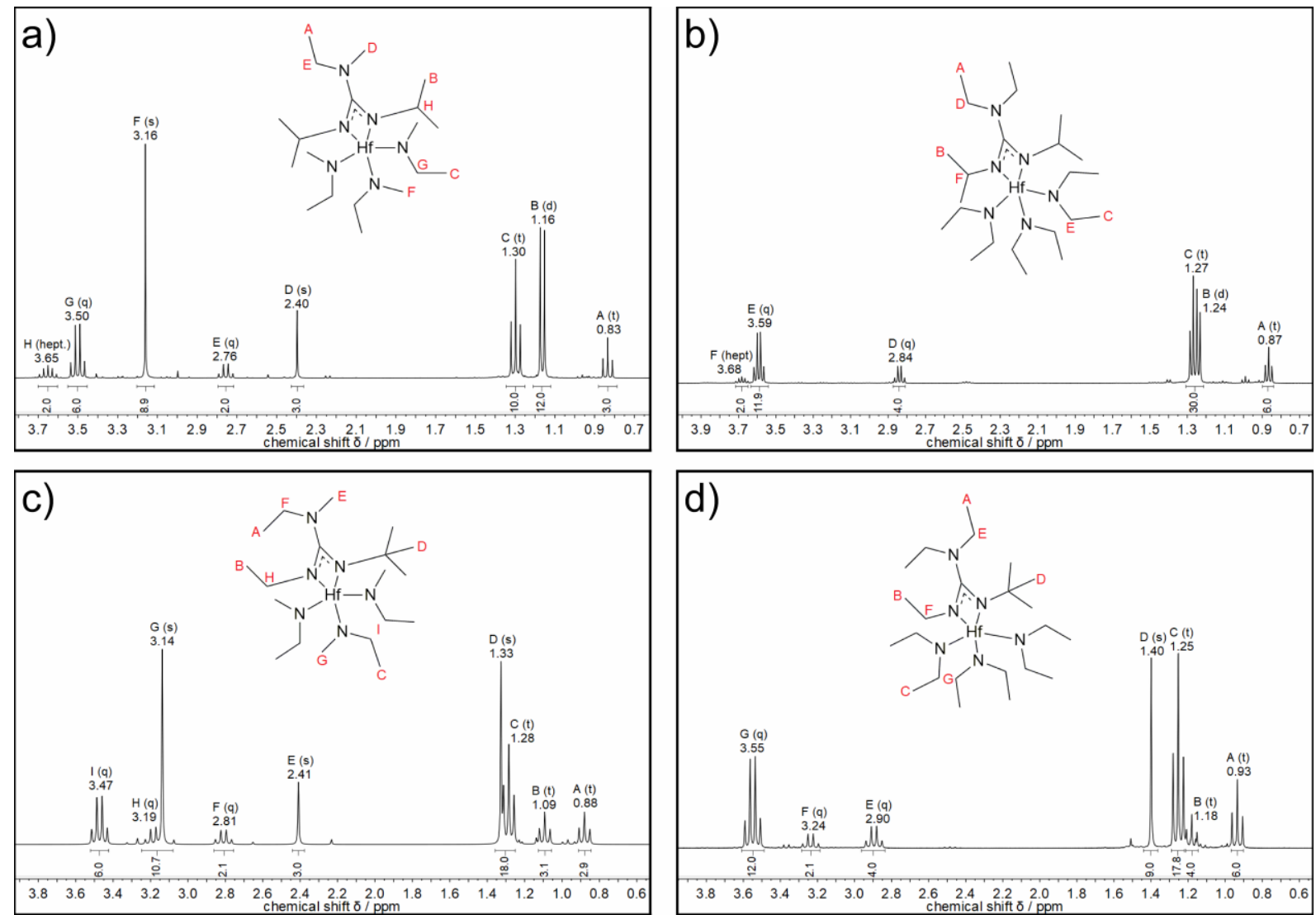

Figure S3: ${ }^{1}$ H-NMR spectra of compound (1) (a), (2) (b), (3) (c) and (4) (d) in d6-benzene.

For compound (1), Figure $\mathbf{S} 3$ a, a triplet $(\mathrm{t})$ with an integral of three can be observed at $\delta=0.83$ ppm correlating to the $\mathrm{CH}_{3}$ protons of the ethyl substituent of the guanidinato backbone nitrogen. The doublet (d) at $\delta=1.16 \mathrm{ppm}$ represents the $\mathrm{CH}_{3}$ protons of the isopropyl groups possessing an integral of 12 . Another triplet signal becomes apparent at $\delta=1.30 \mathrm{ppm}$ and can be assigned to the ethyl $\mathrm{CH}_{3}$ protons of the amido groups. On the higher field side of the spectrum a singlet (s) at $\delta=2.40 \mathrm{ppm}$ can be seen. As it exhibits an integral of three it is allocable to the protons of the methyl group belonging to the backbone nitrogen of the guanidinato ligand. Close by, a quartet (q) with an integral of $\delta=2.76 \mathrm{ppm}$ represents the $\mathrm{CH}_{2}$ protons of the guanidinato backbone nitrogen ethyl substituent. A singlet at $\delta=3.16 \mathrm{ppm}$ with an integral of 9 belongs to the methyl moieties of the amido ligands, while another quartet ( $\delta=3.50 \mathrm{ppm}$ with an integral of six) can be assigned to the $\mathrm{CH}_{2}$ protons of the amido ethyl chains. The heptet (hept.) at $\delta=3.65 \mathrm{ppm}$ with an integral of two originates from the $\mathrm{CH}$ units of the isopropyl groups and completes the spectrum. While for (2), Figure S3 b, a triplet at $\delta=0.87 \mathrm{ppm}$ with an integral of six corresponds to the $\mathrm{CH}_{3}$ moieties of the ethyl side chains attached to the backbone nitrogen of the guanidinato ligand, a quartet with $\delta=2.84 \mathrm{ppm}$ with an integral of four typifies the respective $\mathrm{CH}_{2}$ protons. In a range of roughly $\delta=(1.20-1.30)$ ppm an assembly of signals arises that can be interpreted as multiplet on first sight. Yet, under assistance of temperature-dependent NMR studies that are discussed subsequently, the multiplet appearing at room temperature could be identified as a doublet at $\delta=1.24 \mathrm{ppm}$ overlapping with a triplet $\delta=1.27 \mathrm{ppm}$ resulting in an overall integral of thirty. The contribution of the doublet can be assigned to the terminal $\mathrm{CH}_{3}$ protons of the isopropyl moieties of the guanidinato ligand $(12 \mathrm{H})$, while the contribution of the triplet arises from the terminal $\mathrm{CH}_{3}$ protons of the ethyl substituents of the amido ligands $(18 \mathrm{H})$. In the higher field region, two further signals, the first being a quartet at $\delta=3.59 \mathrm{ppm}$ with an integral of 12 representing the 
$\mathrm{CH}_{2}$ protons of the ethyl side chains of the amido moieties and the second being a heptet at $\delta=3.68 \mathrm{ppm}$ with an integral of two matching the $\mathrm{CH}$ protons of the isopropyl groups, complete the spectrum.

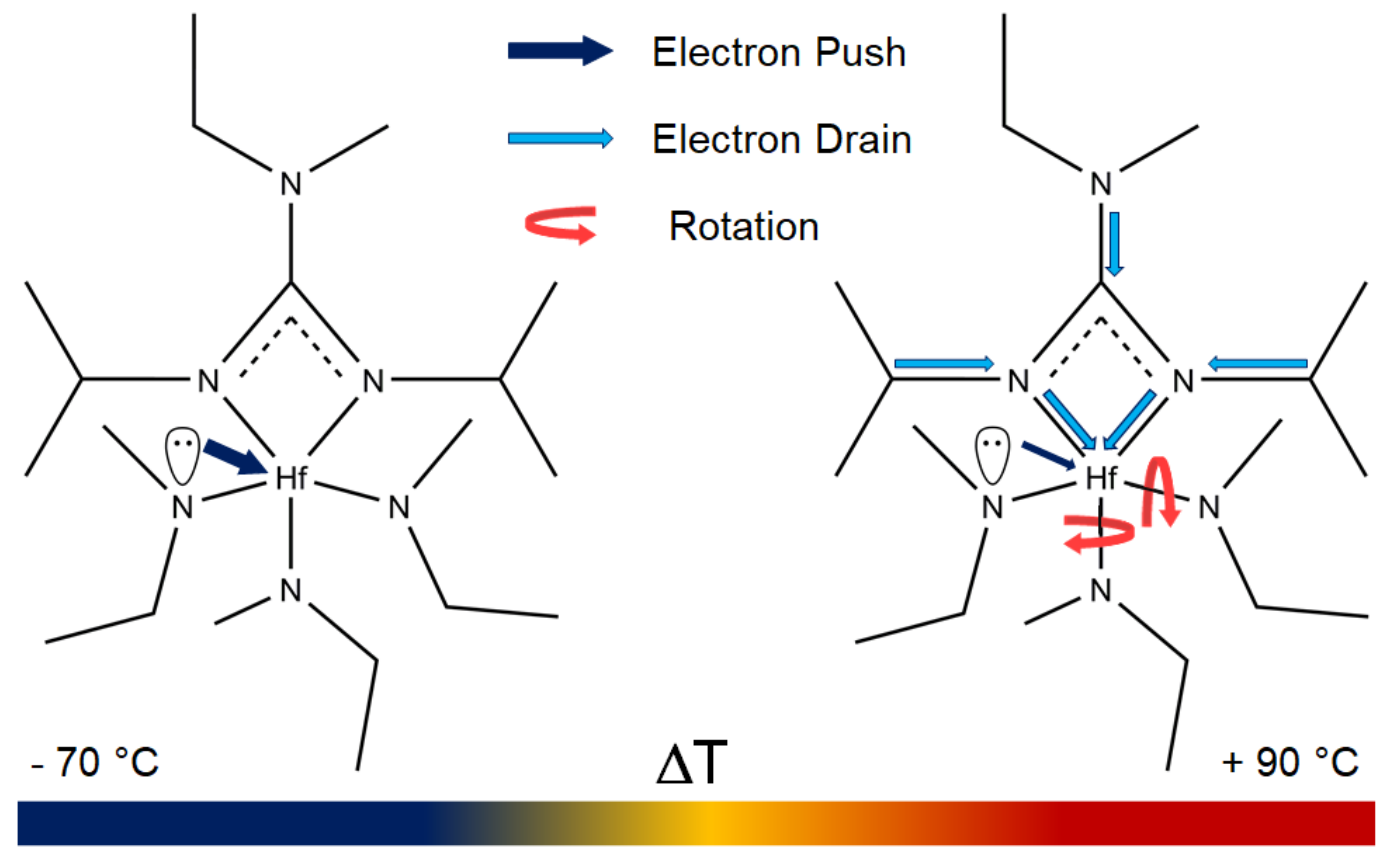

Figure S4: Schematic of temperature induced change of electronic stabilization contributions observed for compound (1) and (2).

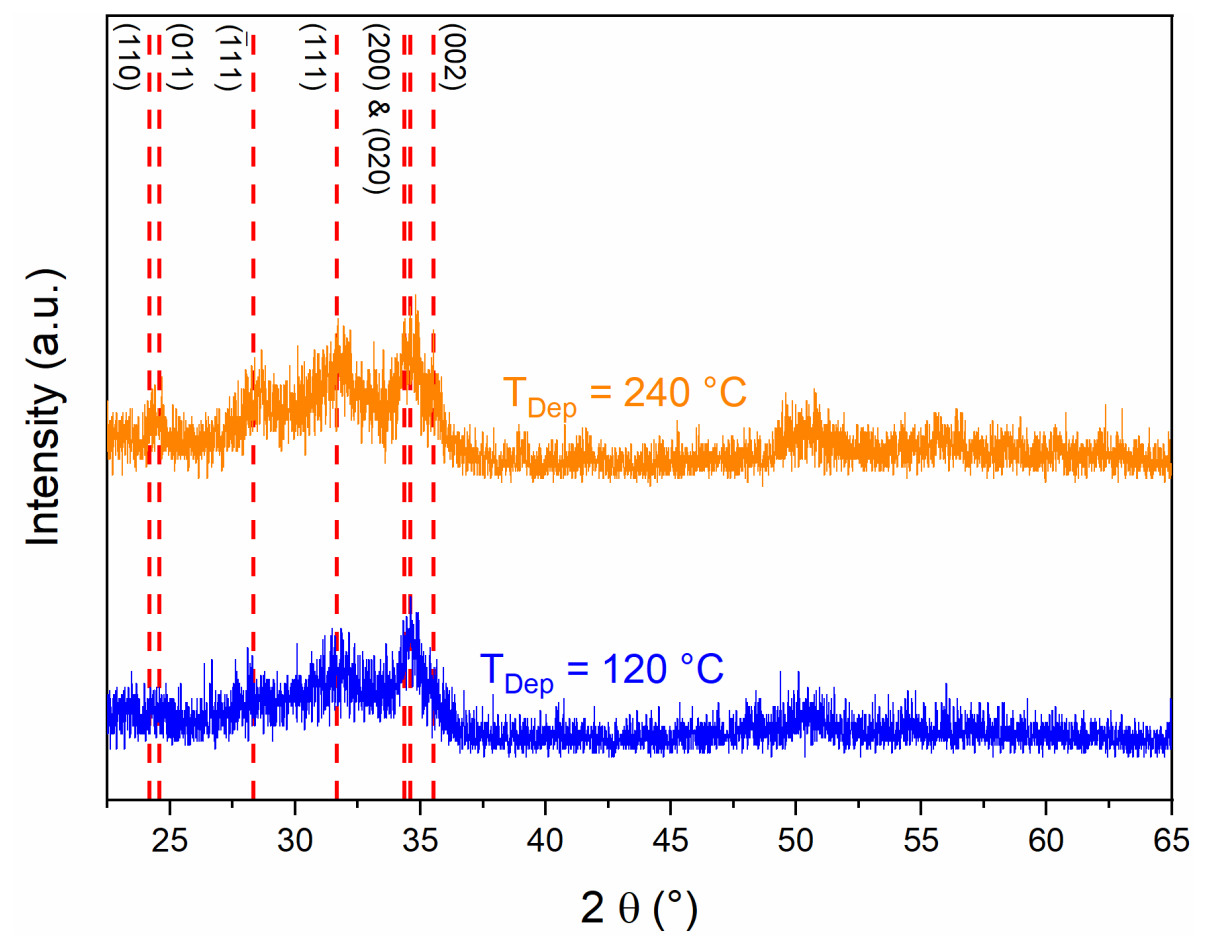

Figure S5: GIXRD of $\mathrm{HfO}_{2}$ thin films with thicknesses of $35 \mathrm{~nm}$ deposited on $\mathrm{Si}(100)$ at $120^{\circ} \mathrm{C}$ (blue) and $240{ }^{\circ} \mathrm{C}$ (orange). The red dotted lines are reference reflexes for monoclinic, crystalline $\mathrm{HfO}_{2}$ (JCP-ID 00-043-1017). 


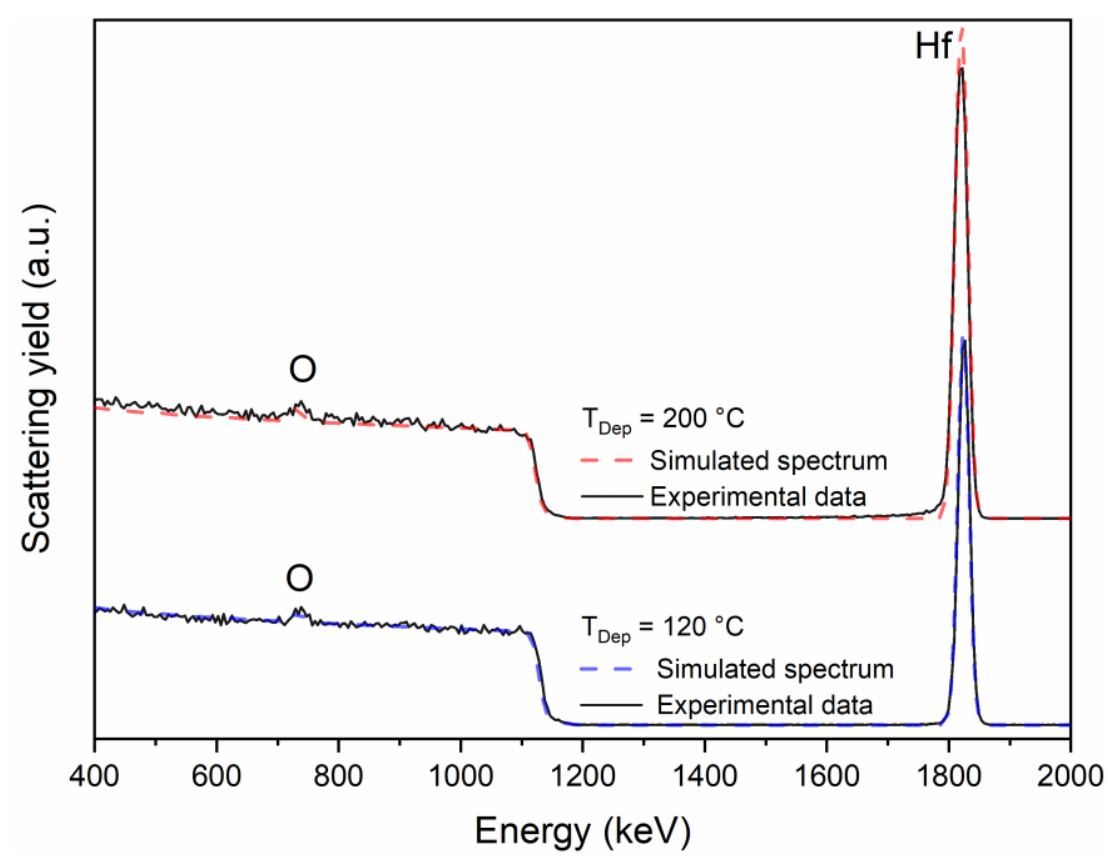

Figure S6: RBS spectra recorded for $\mathrm{HfO}_{2}$ films deposited on $\mathrm{Si}(100)$ at $120^{\circ} \mathrm{C}$ (blue fit) and $200{ }^{\circ} \mathrm{C}$ (red fit). The fits are depicted as dotted lines for clarity.

Table S2: Composition of $\mathrm{HfO}_{2}$ thin films deposited on $\mathrm{Si}(100)$ with varied precursor and plasma pulse lengths using $\left[\mathrm{Hf}\left\{\eta^{2}-(\mathrm{PrN})_{2} \mathrm{CNEtMe}\right\}(\mathrm{NEtMe})_{3}\right](\mathbf{1})$ and $\mathrm{O}_{2}$-plasma at different deposition temperatures.

\begin{tabular}{cccccccc} 
Temperature & Precursor pulse & Plasma pulse & \multicolumn{5}{c}{ Concentration $(\text { at. \%) })^{\text {a) }}$ and O to Hf ratios } \\
\cline { 5 - 7 } & length $(\mathrm{ms})$ & length $(\mathrm{ms})$ & $\mathrm{C}$ & $\mathrm{N}$ & $\mathrm{O}$ & $\mathrm{Hf}$ & $\mathrm{O} / \mathrm{Hf}$ \\
\hline 60 & 2000 & 75 & 19.9 & 20.9 & 44.1 & 15.0 & 2.93 \\
60 & 2000 & 150 & 1.3 & 6.1 & 66.8 & 25.9 & 2.58 \\
60 & 2000 & 270 & 0.3 & 4.7 & 67.8 & 27.2 & 2.50 \\
60 & 2000 & 380 & 0.0 & 1.5 & 68.7 & 29.8 & 2.30 \\
60 & 2000 & 500 & 0.0 & 2.1 & 67.4 & 30.5 & 2.21 \\
80 & 2000 & 500 & 0.0 & 2.9 & 66.4 & 30.8 & 2.16 \\
100 & 2000 & 500 & 0.0 & 1.9 & 67.3 & 30.8 & 2.19 \\
120 & 2000 & 75 & 0.0 & 1.3 & 69.0 & 29.6 & 2.33 \\
120 & 2000 & 150 & 0.0 & 1.7 & 69.4 & 28.9 & 2.40 \\
120 & 2000 & 270 & 0.0 & 2.0 & 68.9 & 29.1 & 2.37 \\
120 & 2000 & 380 & 0.0 & 0.4 & 69.7 & 29.8 & 2.34 \\
120 & 2000 & 500 & 0.0 & 0.0 & 67.3 & 32.7 & 2.06 \\
140 & 2000 & 500 & 0.0 & 0.0 & 66.8 & 33.2 & 2.01 \\
160 & 2000 & 500 & 0.1 & 0.0 & 67.7 & 32.2 & 2.10 \\
180 & 2000 & 500 & 0.0 & 0.5 & 65.8 & 33.7 & 1.95 \\
200 & 2000 & 500 & 0.0 & 0.1 & 66.7 & 33.2 & 2.01 \\
220 & 2000 & 500 & 0.6 & 0.0 & 66.8 & 32.7 & 2.04 \\
240 & 2000 & 500 & 0.5 & 0.6 & 65.9 & 33.0 & 2.00
\end{tabular}

a) $0.0=$ not detected species with detection limits for $\mathrm{C}$ and $\mathrm{N}$ of $2.0 \times 10^{15}$ atoms per $\mathrm{cm}^{2}$ and $8.0 \times 10^{15}$ atoms per $\mathrm{cm}^{2}$, respectively. In all cases, a possible error of 1.5 at $\%$ must be assumed. 


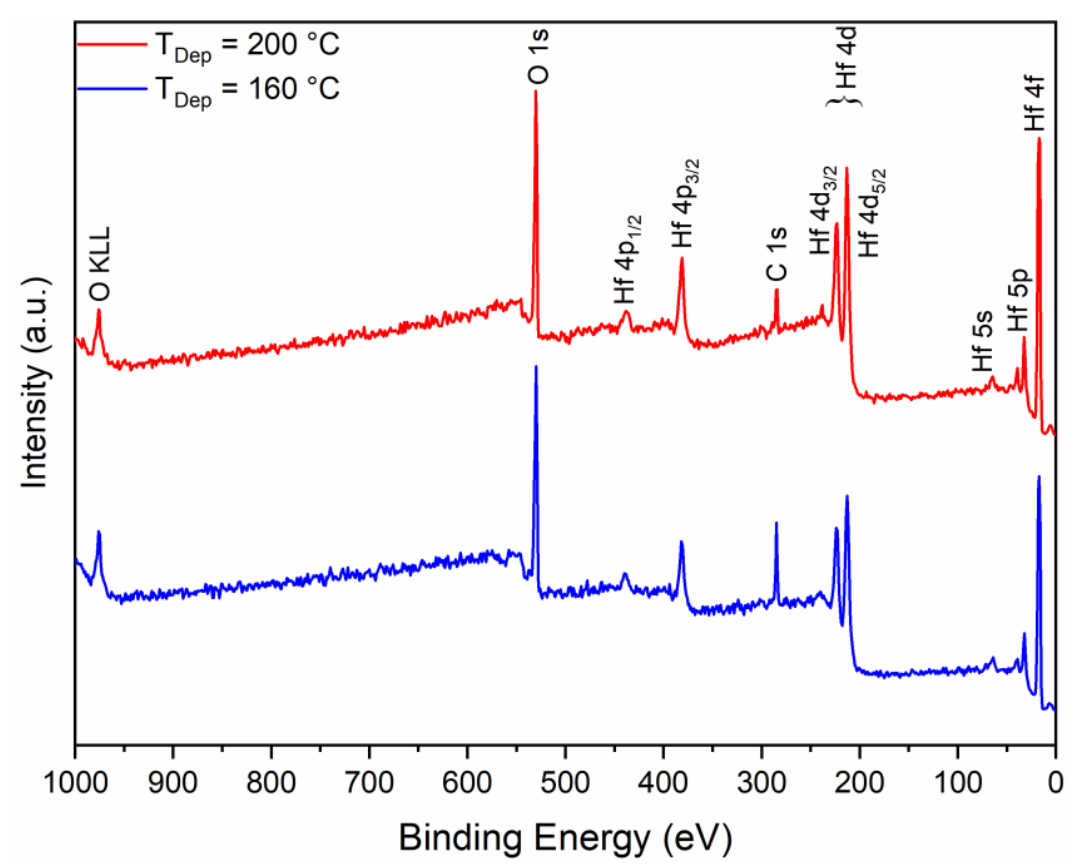

Figure S7: Survey spectra recorded for $\mathrm{HfO}_{2}$ thin films deposited on $\mathrm{Si}(100)$ at $160^{\circ} \mathrm{C}$ and $200^{\circ} \mathrm{C}$. The measurements were conducted at $25^{\circ} \mathrm{C}$ under UHV conditions.

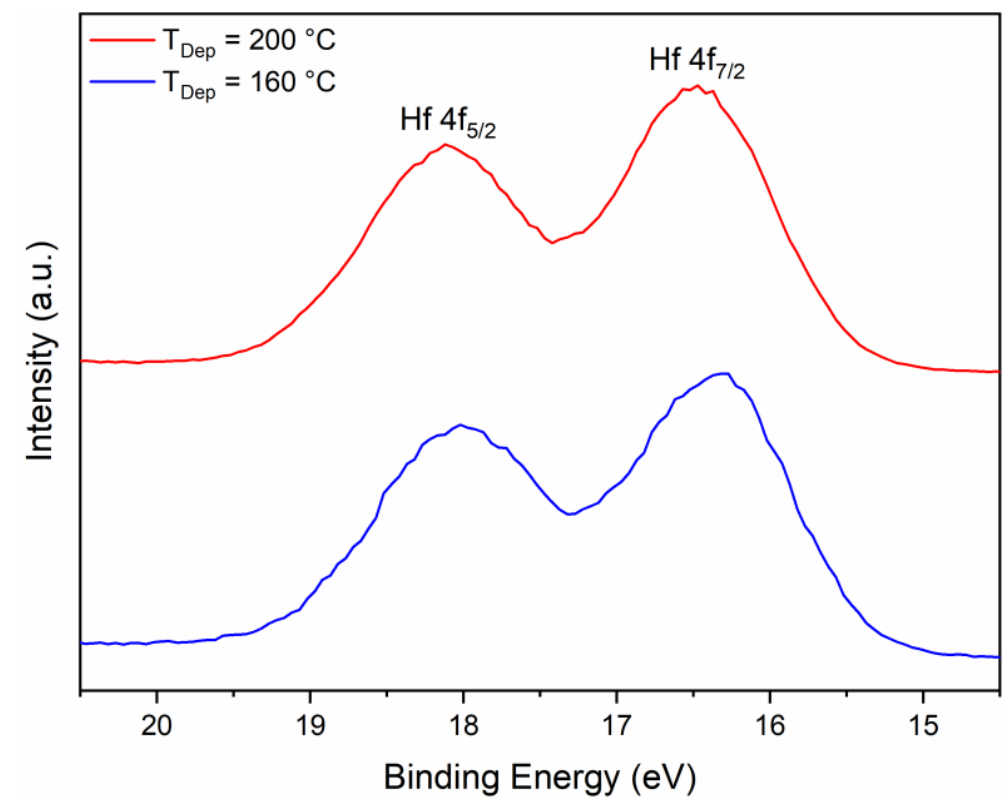

Figure S8: High-resolution XPS spectra of the $\mathrm{Hf} 4 \mathrm{f}$ core level regions recorded for the $160{ }^{\circ} \mathrm{C}$ and $200{ }^{\circ} \mathrm{C} \mathrm{HfO}_{2}$ thin films upon $\mathrm{H}_{2}$ (1 mbar) exposure at $25^{\circ} \mathrm{C}$. 


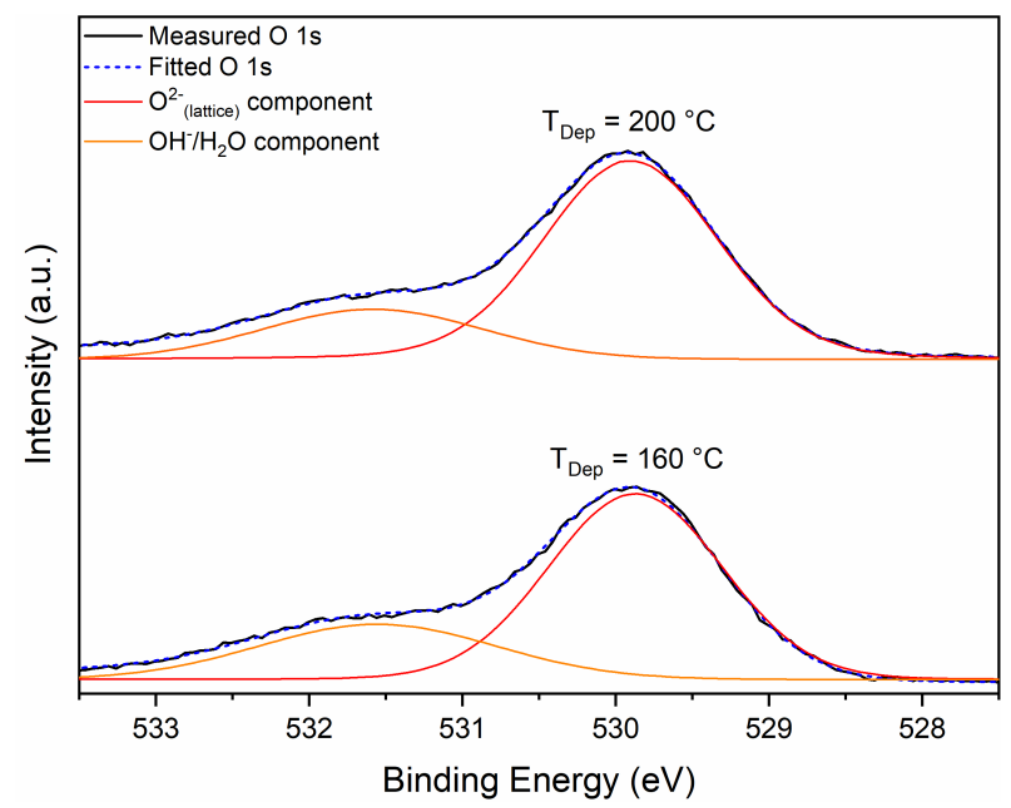

Figure S9: XPS O 1s core level spectra of $20 \mathrm{~nm} \mathrm{HfO}_{2}$ thin films deposited at $160{ }^{\circ} \mathrm{C}$ and $200{ }^{\circ} \mathrm{C}$ on $\mathrm{Si}(100)$ recorded upon $\mathrm{O}_{2}(5 \mathrm{mbar})$ exposure at $25^{\circ} \mathrm{C}$.

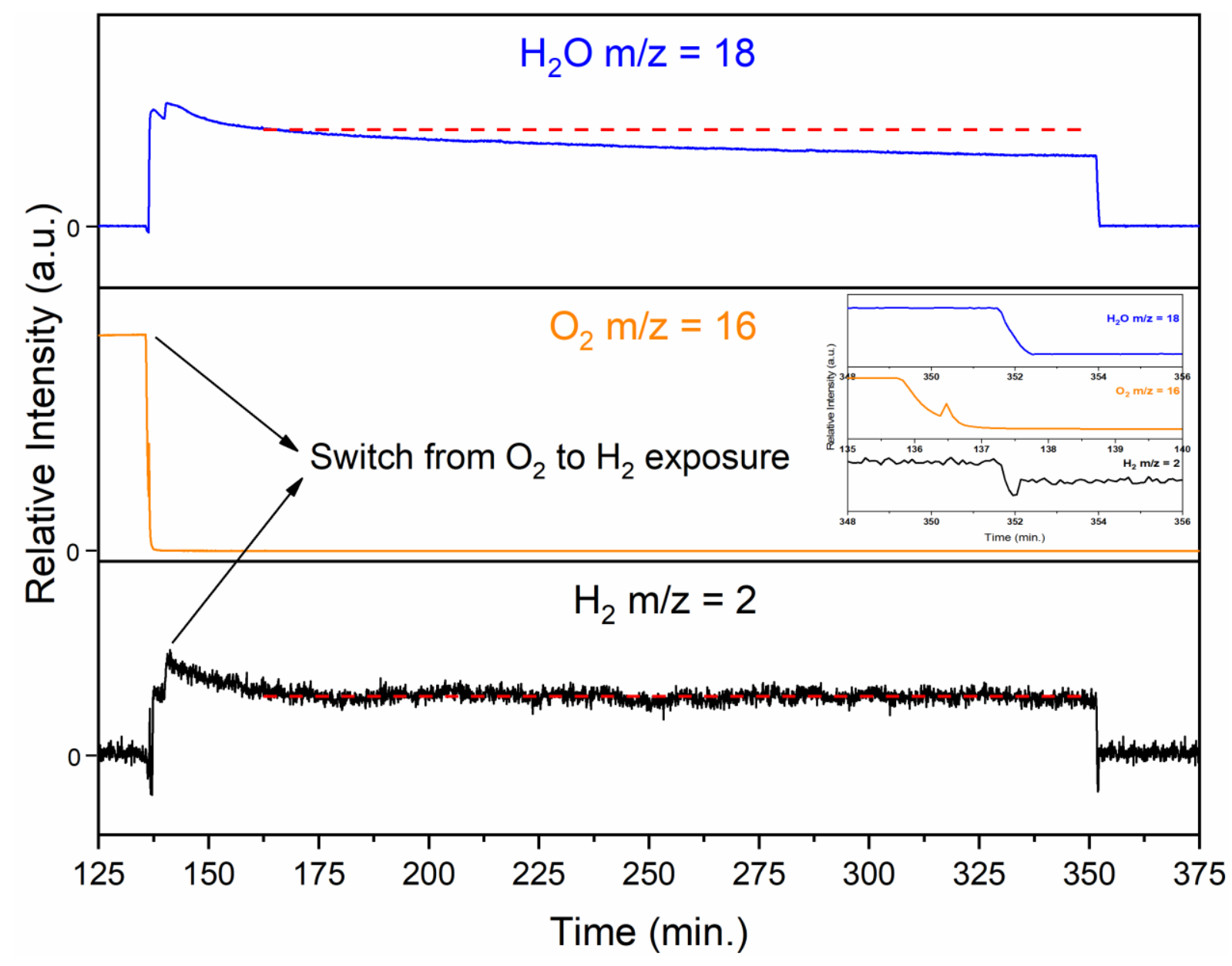

Figure S10: Qualitative mass spectrometry readings for $\mathrm{H}_{2}$ (black trend), $\mathrm{O}_{2}$ (orange trend) and $\mathrm{H}_{2} \mathrm{O}$ (blue trend) recorded during a first $500{ }^{\circ} \mathrm{C}$ long-term exposure experiment for the $160{ }^{\circ} \mathrm{C}$ deposited $\mathrm{HfO}_{2}$ thin film. After initial $\mathrm{O}_{2}$ exposure, the exposure gas was switched to $\mathrm{H}_{2}$ upon which immediate formation of $\mathrm{H}_{2} \mathrm{O}$ was observed. The decline of the $\mathrm{H}_{2} \mathrm{O}$ signal over time can be correlated with the decreasing amount of adsorbed $\mathrm{O}_{2}$ on the $\mathrm{HfO}_{2}$ thin film surface 
whose slow conversion is monitored. Red dotted lines in the charts help to guide the eye. The inset illustrates the slow decay of $\mathrm{H}_{2} \mathrm{O}$ formation with decaying $\mathrm{H}_{2}$ exposure.

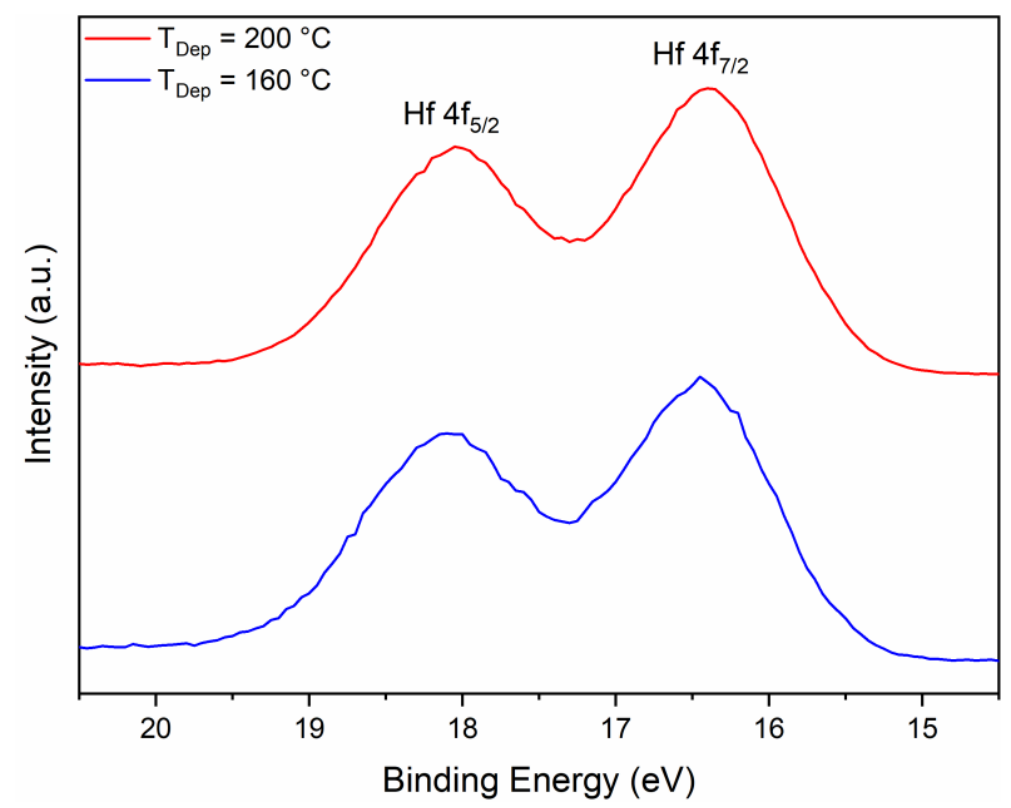

Figure S11: High-resolution XPS spectra of the $\mathrm{Hf} 4 \mathrm{f}$ core level regions recorded for the $160{ }^{\circ} \mathrm{C}$ and $200{ }^{\circ} \mathrm{C} \mathrm{HfO}_{2}$ thin films after several exposure sweeps to $\mathrm{O}_{2}(5 \mathrm{mbar})$ and $\mathrm{H}_{2}(1 \mathrm{mbar})$ at $500{ }^{\circ} \mathrm{C}$.
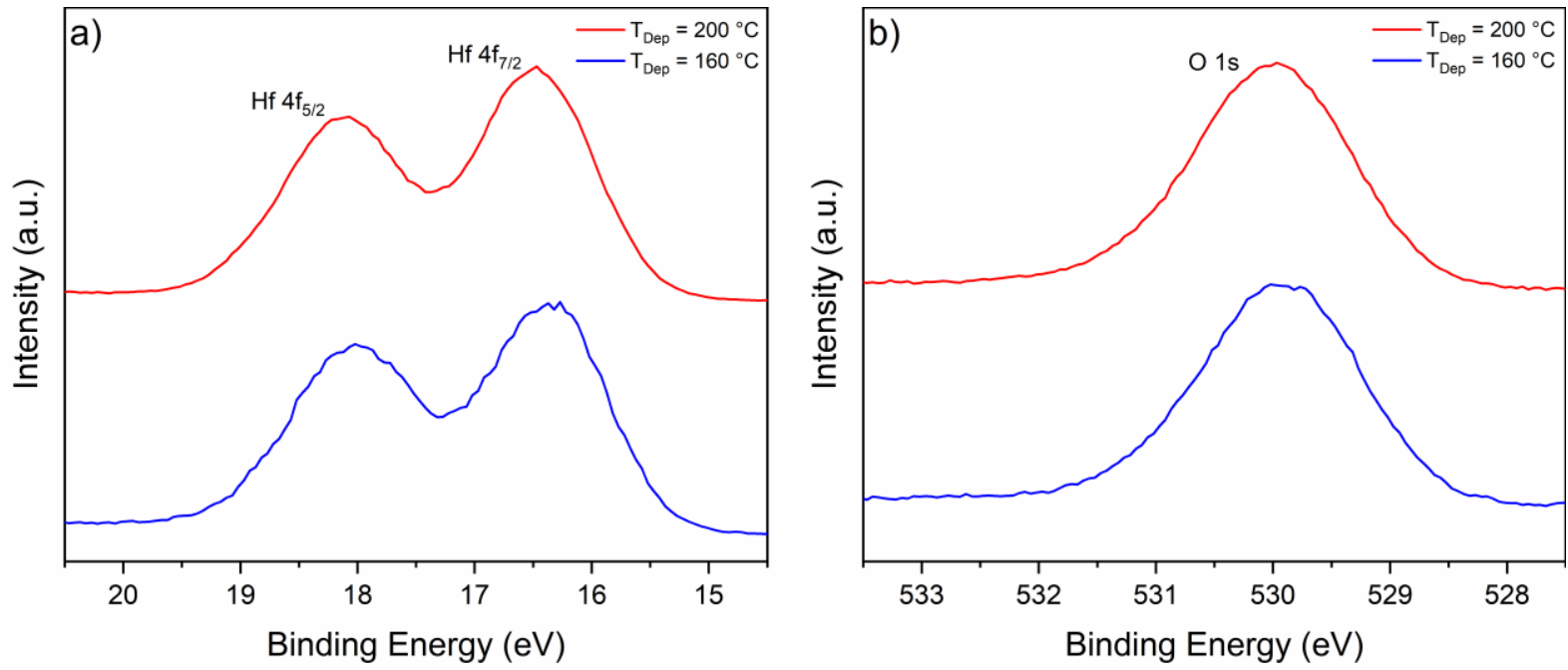

Figure S12: High-resolution XPS spectra of $20 \mathrm{~nm} \mathrm{HfO} 2$ thin films deposited at $160^{\circ} \mathrm{C}$ and $200{ }^{\circ} \mathrm{C}$ on $\mathrm{Si}(100) \mathrm{recorded}$ in UHV at $500{ }^{\circ} \mathrm{C}$ showing a) the $\mathrm{Hf} 4 \mathrm{f}$ and b) the 01 s core level regions of the different films. 


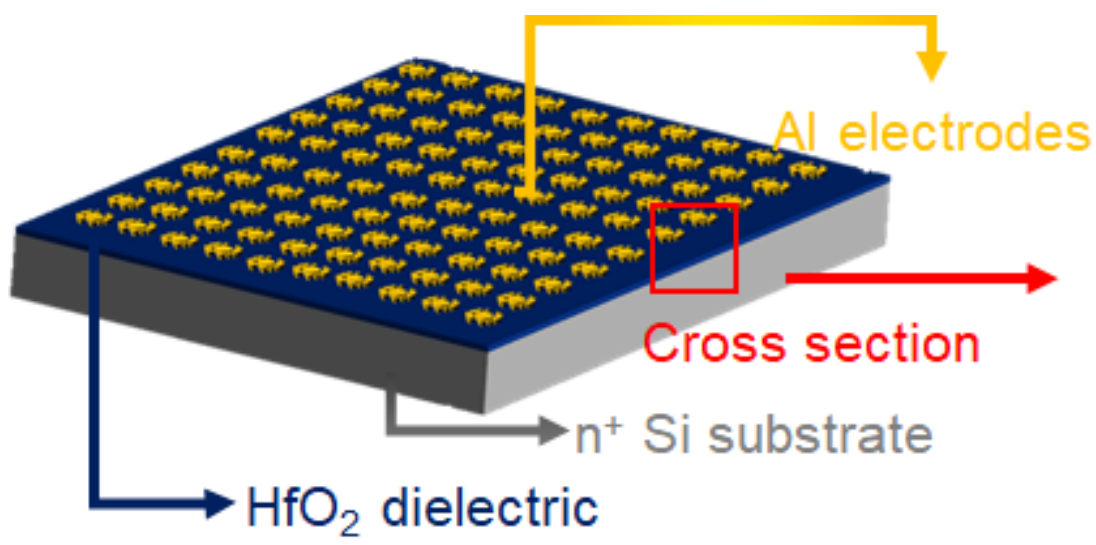

Figure S13: Schematic illustration of the metal insulator semiconductor (MIS) capacitor structures fabricated for the assessment of $\mathrm{HfO}_{2}$ dielectric properties. 